\title{
FM Broadcasting Exposure Time and Malignant Melanoma Incidence
}

\author{
ÖRJAN HALLBERG ${ }^{1}$ AND OLLE JOHANSSON ${ }^{2}$ \\ ${ }^{1}$ Polkavägen 14B, 14265 Trångsund, Sweden \\ ${ }^{2}$ Experimental Dermatology Unit, Department of Neuroscience, \\ Karolinska Institute, S-171 77 Stockholm, Sweden
}

\begin{abstract}
Object: To analyze the age-specific incidence of malignant melanoma in Sweden since 1958 in order to see if the reported general increase in incidence would be explained by a sudden exposure to an environmental stress to the population. Methods: Incidence data for all age groups was collected from the Swedish National Board of Health and Welfare databases for each year between 1958 and 2002. The incidence in all 288 municipalities of Sweden was correlated to the number of FM transmitters covering each municipality. Results: The age-specific incidence was found to be constant over the last 20-30 years for people younger than 50 years while the incidence for older age groups still are constantly increasing. The total incidence in different municipalities was found to be a strong function of the number of covering FM transmitters. Conclusions: The age-specific incidence of malignant melanoma of the skin appears to be following a pattern of response to an imposed environmental change in 1955. We believe that the frequency modulation (FM) broadcasting radiation at whole-body resonant frequencies is such an environmental stress.
\end{abstract}

Keywords Broadcasting; Exposure time; FM; Malignant; Melanoma; Radiation.

\section{Introduction}

The incidence and mortality rates of malignant melanoma are increasing rapidly in many countries in the world [1-3]. The traditional explanation for this increase has been excessive sunburn and increased travel [3]. We have presented data that associate this increase with the exposure of the population to whole-body resonant electromagnetic fields, such as frequency modulation (FM) broadcasting around $100 \mathrm{MHz}$ [4].

Address correspondence to Örjan Hallberg, Polkavägen 14B, Trångsund 142 65, Sweden; E-mail: oerjan.hallberg@swipnet.se 
We have also demonstrated that an abrupt increase in mortality from melanoma of skin was a fact several years before any large-scale tourist sun-travels began in Sweden [5].

In order to better characterize the change in melanoma incidence over time, we analyzed the age-specific incidence in Sweden for all years since 1958. The purpose of this analysis was to see if earlier results based on data starting from 1975 would also hold from 1958, the first set of data available to us [4].

\section{Methods}

Data on cancer incidence is available without charge from the Swedish National Board of Health and Welfare [6]. We collected age-specific incidence data year by year from 1958 to 2002. Data was provided in age steps of 5 years, i.e., 0-4 years of age, 5-9 years of age, etc., for every year 1958-2002.

We have previously noted a strong relationship between the number of FM transmitters that covered a municipality and its melanoma incidence [4]. In order to visualize this relationship we calculated the median incidence of municipalities covered by in average $0-5$ transmitters in steps of 0.25 if relevant. This might be the case when 2 transmitters covered $75 \%$ of the area and 3 transmitters covered $25 \%$, with the average number of transmitters 2.25 for that municipality.

\section{Results}

Figure 1 gives the measured age-specific incidence of malignant melanoma in Sweden between 1958 and 2002.

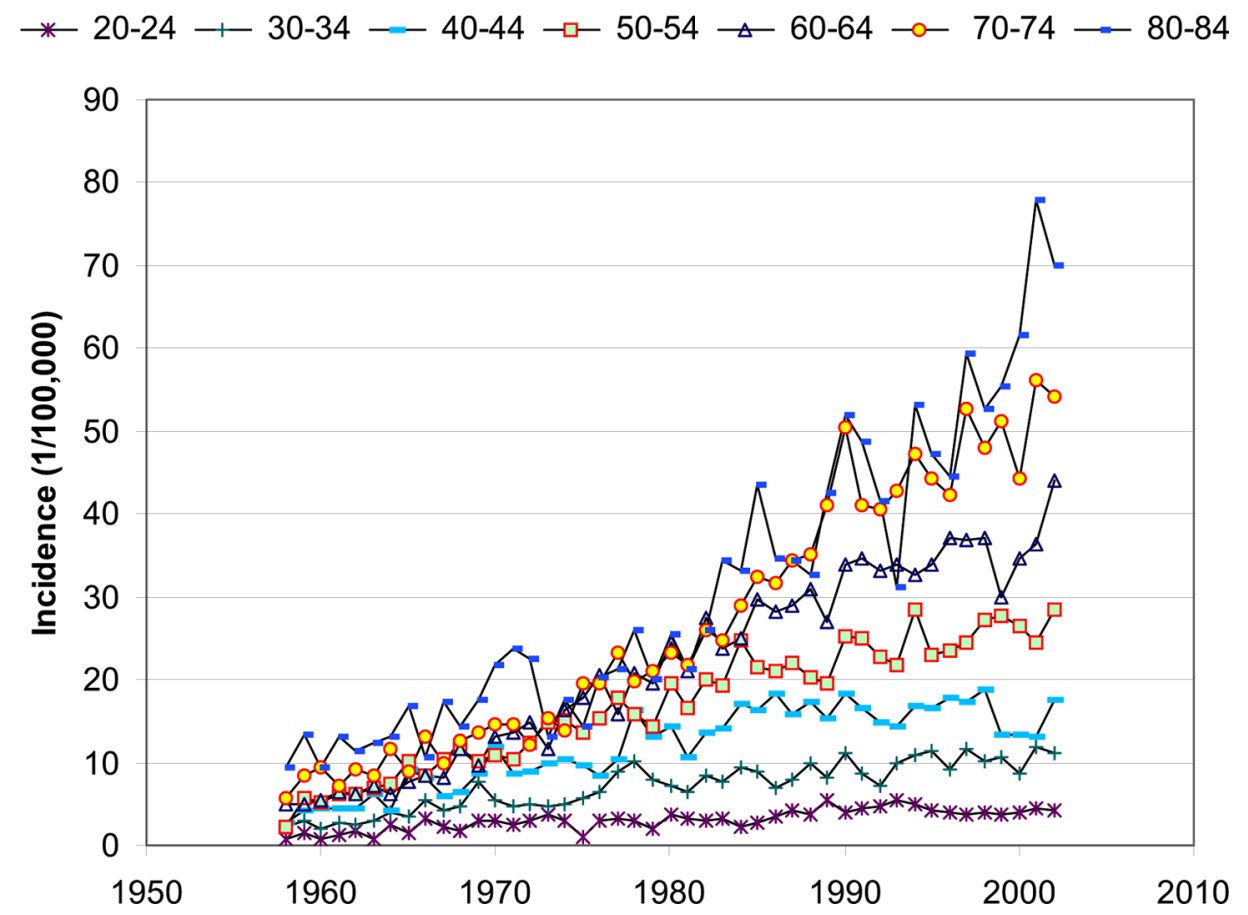

Figure 1. Age-specific incidence of malignant melanoma in Sweden from 1958-2002. 
Figure 1 shows that the incidence levels off and has been quite stable for many years for the younger age groups. The age group 20-24 years has been stable since about 1967, the age group 30-34 has been stable since 1977, the age group 40-44 has been stable since 1985, and the age group 50-54 has possibly leveled off from 1995. All remaining age groups are still increasing and do not seem to have reached their final, stable level yet. The incidence for older individuals is thus expected to increase further until we will see a leveling off similar to the younger age groups.

Figure 2 gives the incidence vs. age as measured in 1958 and as averages for the measurements in the time period 1999-2002.

Hallberg and Johansson demonstrated how an exposure time-specific incidence was extracted from reported incidence data and from the reported increase of the FM-broadcasting networks in Sweden, Norway, Denmark, and USA [4]. In the same paper an exposure time-specific incidence was also estimated directly from the age-specific incidences (ASIs) reported in 1996 when time zero was defined as 12 years of age (puberty age). These estimates are shown in Fig. 3 as accumulated incidence together with the corresponding data as derived from Fig. 2. All three independently calculated estimates came up with basically the same cumulative density function, while the one based on age-specific incidence data reported in 1958 is different and much lower.

The number of FM transmitters covering each municipality and its melanoma incidence were analyzed. The median incidence $(1 / 100,000)$ for municipalities grouped by the number of transmitters covering the municipality was calculated. See Table 1 and Fig. 4.

\section{Discussion}

The accumulated incidence shown in Fig. 3 can be used to model the risk of catching malignant melanoma as a function of exposure time from FM-broadcasting radiation. Table 2 gives a list of log-normal distribution parameters based on different sets of data.

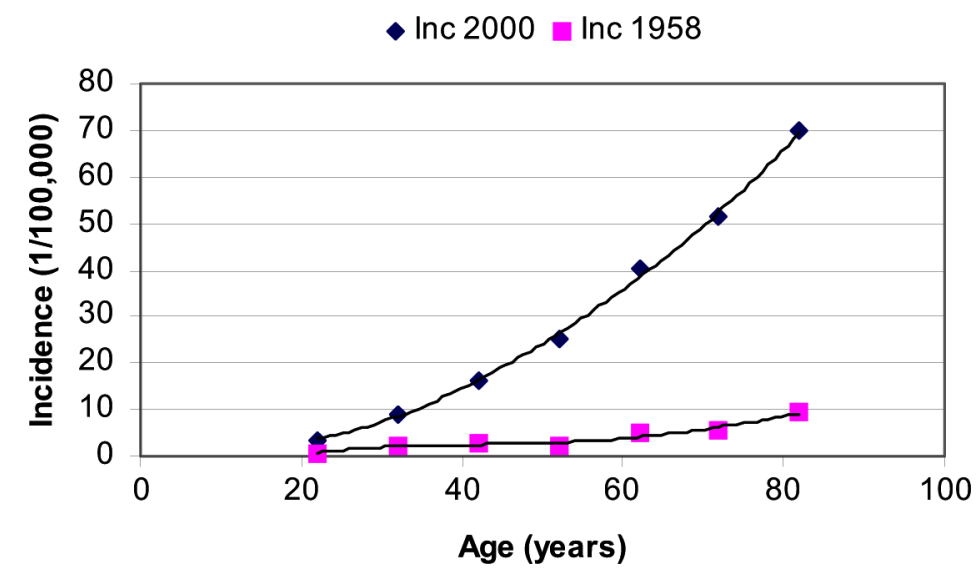

Figure 2. The graph gives incidence vs. age as measured in 1958 and as averages for the time period 1999-2002 for respective age groups all here noticed as Inc 2000. 


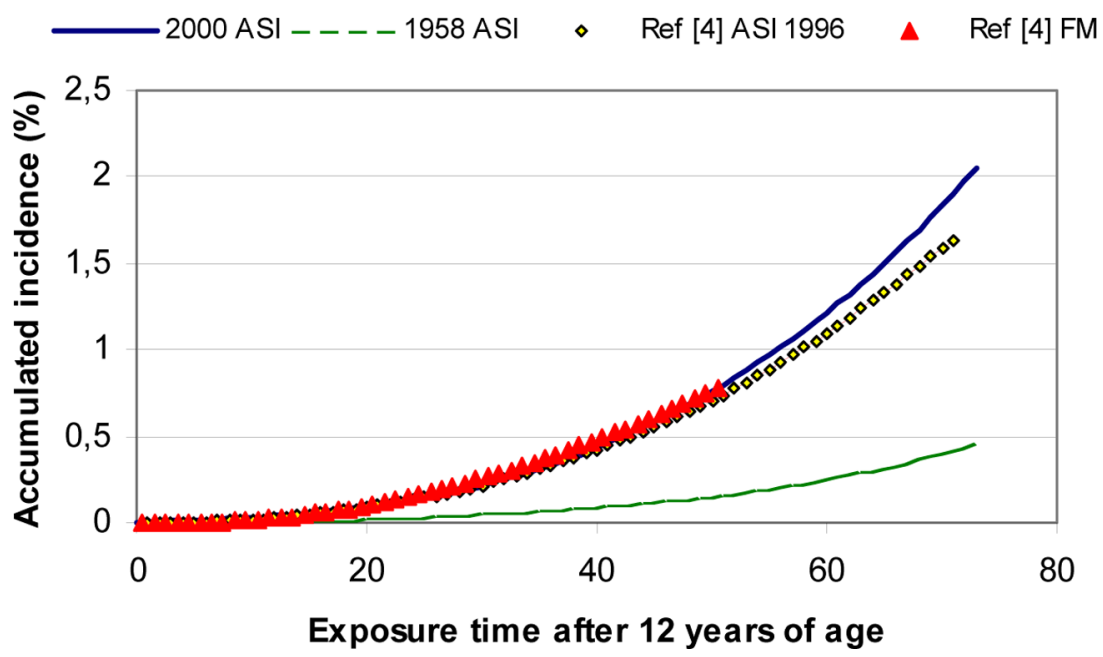

Figure 3. The accumulated incidence of melanoma of skin was estimated from 4 different sets of data.

The cumulative density function (cdf) plots based on ASI-data and the Swedish FM-data are plotted in Fig. 3. All of the curves are basically identical except for the one based on ASI-data from 1958. The data may also be modeled by a normal distribution. Such a model gave a median life of 200 years and a standard deviation of 62 years for the exposure-time-specific incidence. Before 1958 the normal distribution had corresponding parameters of 251 years and 68.7 years. The age-specific incidence over time can be theoretically calculated from these statistical distributions.

\section{Table 1}

The municipalities grouped by the average number of FM transmitters that are covering each municipality

\begin{tabular}{lcc}
\hline Transmitters & Median incidence & Number of municipalities \\
\hline 0 & 2 & 288 \\
1 & 6 & 22 \\
1.25 & 10 & 11 \\
1.5 & 10 & 17 \\
2 & 12 & 39 \\
2.25 & 12.5 & 9 \\
2.5 & 15 & 29 \\
2.75 & 15 & 7 \\
3 & 17 & 90 \\
3.25 & 17.5 & 8 \\
3.5 & 19 & 17 \\
4 & 19.5 & 23 \\
4.5 & 22 & 5 \\
\hline
\end{tabular}




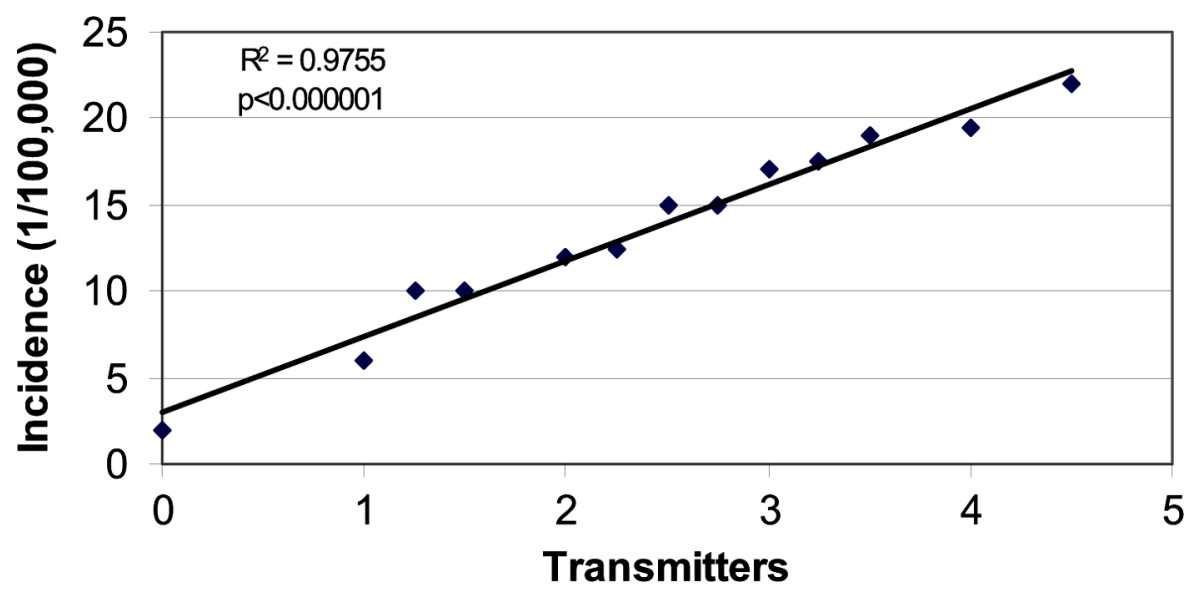

Figure 4. The graph gives the median melanoma incidence vs. the average number of FM transmitters that are covering each municipality. The incidence at 0 transmitters represents the status in all of Sweden before 1955. Each group contains 5 or more municipalities.

By using such a life distribution together with available information on the number of exposed people at different points in time, the total number of melanoma cases can be estimated. The method used for this calculation was described previously [4].

Table 2

The table gives distribution parameters for an exposure time-depending accumulated incidence of malignant melanoma of the skin based on different sets of data and a log-normal distribution

Data set

Dispersion (base 10)

Time to $0.1 \%$

Age-specific incidence 2000

Age-specific incidence 1996 [4]

0.6

0.55

0.57

0.59

0.55

20.72 years

Annually FM-exposed people and reported new melanoma cases in Denmark [4]

Annually FM-exposed people and reported new melanoma cases in USA [4]

Age-specific incidence 1958 and reported new melanoma Annually FM-exposed people

and reported new melanoma cases in Norway [4] acc. incidence 
Figure 1 shows that malignant melanoma in 1958 was not as common as it is today, with an incidence less than 10/100,000 per year even for the oldest age groups. By time this level was rising to today's level of about 80/100,000 (i.e., eight times higher) for the older age groups. We noticed a similar type of increase in age-specific mortality by time for other countries including Norway, Denmark, and Switzerland.

Another observation is that the incidence for age groups up to 50-54 years now is not increasing at all. Figure 1 shows that the general increase in incidence is due to the increased melanoma incidence among the elderly. The graph also indicates that we will not see a constant incidence among those older age groups until they consist of people born in 1946 or later.

All age groups that have been exposed to FM-radiation since the time of puberty seem to have a constant incidence of skin melanoma, independent of which year the measurement was done. This is contrary to the concept that melanoma incidence is increasing due to increasing sun exposure. Our data suggests that this is not the only reason for the increase. The incidence is not increasing within each individual age group that has had the time to stabilize. What makes the agestandardized rates (ASR) increase over time is that older age groups have not yet stabilized. There are no 80 -year-old persons who have been exposed for FMbroadcasting radiation since puberty, and this will not happen until 2025.

Figure 4 shows that the number of FM transmitters covering a municipality is an important factor behind its melanoma incidence. It should be pointed out that a positive correlation between two variables does not prove a causal link and may be misleading if not supported by other data. In this case it is supported by the fact that a mathematical function describing the accumulated incidence vs. exposure time can be used to calculate the number of annual new cases of malignant melanoma in several countries, as has been done for Sweden, Norway, Denmark, and the USA, with accuracy [4]. This appears to be the same for the USA where FM-broadcasting did not show a major increase until 1974. The other three countries all started earlier, around 1955.

Many variables can be confounding influences. In Sweden the incidence of melanoma of skin is decreasing by increasing latitude (or decreasing UV-index). On the other hand the general trend in Europe is that the incidence is increasing by latitude. In France it is increasing; in Denmark it is unrelated; and in Sweden, Finland, and Norway it is decreasing by latitude. In all investigated countries the incidence is increasing with the number of covering FM-transmitters, though. Melanoma incidence is also correlated with population density. In Sweden it has a positive correlation to population density, while in England it has a negative correlation.

Our hypothesis is that full-body resonant electromagnetic fields (EMFs) may disturb cell repair mechanisms and the immune system. Such a resonance is likely to give the largest fluctuations of body potential on the surface of the middle parts of the body, i.e. the chest, trunk, back, upper parts of the legs, etc. This is also where melanoma of skin most predominantly is found, not on the hands or on other parts of the body much more exposed to the sun light. If a place is covered by more than one FM-transmitter the probability of sleeping in a resonant direction will increase, as shown in Fig. 4.

Stress hormones are affected by low-level radio-frequency (RF) EMFs, according to Vangleova et al. [7]. A good review of biological effects of high- and 
extremely high-frequency EMFs was done by Mileva et al. [8]. Boscolo et al. demonstrated that the immune system of women is affected by radio television broadcasting towers [9]. Workers exposed to EMFs and polychlorinated biphenyls were compared regarding prostate cancer mortality by Charles et al. [10].

Finally, it is known that the skin acts as a low-voltage battery of about $10-20 \mathrm{mV}$ [11]. After a wound, blood shorts this battery and an electric current supports the transport of new cells to heal the wound. We believe that such cell repair mechanisms, including those related to malignancy, can be affected by externally applied EMFs, either low-frequency or body-resonant ones.

Most of the FM transmitters emit horizontally polarized fields. This means that while sleeping, an individual may be placed in resonance by their position if the bed is directed toward an FM-broadcasting main transmitter. It is also known that many cell repair functions are done by the body at night by hormones such as melatonin. A continuous disturbance of this process over many years may increase cancer risks, including those other than skin cancer.

According to our hypothesis the effect of the number of surrounding transmitters would be much stronger than from the average transmitted power density over an area. Such a calculation of the power density was done for the 289 municipalities of Sweden. There appeared to be no noticeable correlation between melanoma incidence and radiated power density. A closer analysis of melanoma incidence showed, however, that the high incidences were found in areas covered by many FM towers while the low incidences were found in areas covered by only one transmitter [4].

The effect of increased tourism and sun-travels in Sweden found no convincing relationship [5]. In October 2004 this reference was the only hit in PubMed when searching for body-resonant electromagnetic fields. No laboratory or animal experiments have to our knowledge been reported where the effect of body-resonant weak fields has been studied.

\section{Conclusions}

Malignant melanoma is an example of a disease that was very rare before 1955 but has increased dramatically over the past 50 years. This increase may be due to an environmental agent which interferes with cell repair mechanisms normally active during sleep. We have shown that an environmental change of this type might be the exposure of a population to whole-body resonant EMFs via FM broadcasting transmitters [4, 5]. In Sweden FM broadcasting started in 1955 and had increased considerably by 1957 .

Melanoma incidence has stayed constant for many years for age groups 20-24, 30-34, and 40-44, which may argue against the hypothesis that an increased exposure to UV-radiation from the sun is the main reason for the increasing ASI. Instead, all data points to an exposure time-dependent incidence, and that exposure started in the mid-1950s in Sweden.

Similar types of analysis should be done also for other neoplasms such as lung, breast, bladder, and others. Since the hypothesis is that horizontally polarized FM broadcasting is weakening our immune defense system during sleep at night, one intervention might be to shut down FM broadcasting transmission at night. Today, most transmitters are broadcasting at full power even if no program is being sent just to save manpower costs for the start up of transmitters in the mornings. 


\section{Acknowledgments}

The authors want to acknowledge the support from the Karolinska Institute and the Cancer and Allergy Foundation.

\section{References}

1. Ashton, J.F.; Laura, R.S. Environmental factors and the etiology of melanoma. Cancer Causes Control. 1993, 4, 59-62.

2. Moan, J.; Dahlback, A. The relationship between skin cancers, solar radiation and ozone depletion. Br. J. Cancer 1992, 65, 916-921.

3. Osterlind, A. Epidemiology on malignant melanoma in Europe. Acta Oncol. 1992, 31, 903-908.

4. Hallberg, Ö.; Johansson, O. Melanoma incidence and frequency modulation (FM) broadcasting. Arch. Envir. Health 2002, 51, 32-40.

5. Hallberg, Ö.; Johansson, O. Malignant melanoma of the skin-not a sunshine story! Med. Sci. Monit. 2004, 10, CR336-340.

6. The National Board of Health and Welfare, 2004, www.sos.se.

7. Vangleova, K.; Israel, M.; Mihaylov, S. The effect of low level radiofrequency electromagnetic radiation on the excretion rates of stress hormones in operators during 24-hour shifts. Cent. Eur. J. Publ. Health 2002, 10, 24-28.

8. Mileva, K.; Georgieva, B.; Radicheva, N. About the biological effects of high and extremely high frequency electromagnetic fields. Acta. Physiol. Pharmacol. Bulg. 2003, 27, 89-199.

9. Boscolo, P.; Di Sciascio, M.B.; D’Ostilio, S.; Del Signore, A.; Reale, M.; Conto, P.; Bavazzano, P.; Paganelli, R.; Di Gioacchino, M. Effects of electromagnetic fields produced by radiotelevision broadcasting stations on the immune system of women. Sci. Total Environ. 2001, 273, 1-10.

10. Charles, L.E.; Loomis, D.; Shy, C.M.; Newman, B.; Millikan, R.; Nylander-French, L.A.; Couper, D. Electromagnetic fields, polychlorinated bisphenyls, and prostate cancer mortality in electric utility workers. Am. J. Epidemiol. 2003, 157, 683-691.

11. Nuccitelli, R. Non-invasive measurement of the electric fields in mammalian skin wounds, Proc. Biol. effects of EMFs, Kos, Greece, 4-8 Oct. 2004, p. 438. ISBN 960-233$151-8$. 